\title{
Die Rotfäule.
}

\author{
Bon Forftmeifter Marl Maner in Sthongn.
}

(ร)แนnf.)

III. Dá̧ Die Faulfolyftatiftit, wie fie hier betrieben wurbe, nidit voll befriedigt, ift flar. Der llmitand, daßj Szart= und Wetchböben viel= fach ineinander übergehen, ift fïr bie Statiftif ftörend und hemmend.

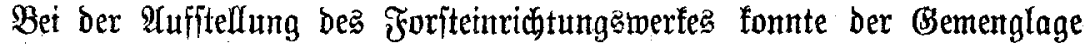
Der $\mathfrak{Y o ̈ b e n ~ n i d j t ~ v o l l ~ R e c h , n u n g ~ g e t r a g e n ~ w e r b e n , ~ w e n n ~ a l f z u v i e l ~ D e t a i l ~}$ vermieden werben wollte. $\mathfrak{U}$ ț werDen, Daß̧ eine föhärfere Trentung Der Unterabteilungen nach Böben,

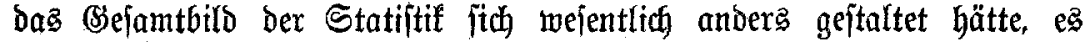

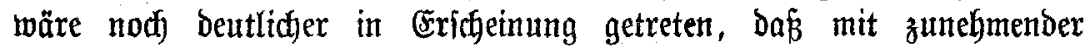
Feurthtigfeit des Boden

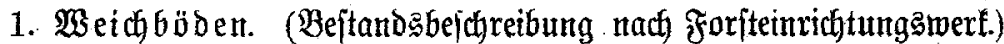

I. 2d. Szöhe 720-724 m. Sage und Boden: Șm Sïb=Teile geringe, wellenförmige (Frbebung aus der Fflachmoorumgebung; Gier fanbiger Lebmboden teilweije zählettig; im übrigen Flachmoorboben, bein über= gang vom Mineralboden zum Moorboben lettige Unterlage (nẩ). $\mathfrak{g}_{\mathfrak{g}} \mathfrak{z}_{z}=$ art: Fichte $\frac{66+110}{77}$ jähria.

Bon $242 \mathrm{fm} 16 \mathrm{fm}$ faul $=6 \%$ Faulgolz.

I. 10b. Şähe $728 \mathrm{~m}$. Booden: Fllachnoorboden von ziemlicher Tieje Der Mooridicht; ziemlich zeriegt, teilmeije entwäffert, vielfach in Moor= erde übergeheno, auf flemer Fläht) Zwifichenmoorcharafter.

Bon $234 \mathrm{fm} 12 \mathrm{fm}$ faul $=5 \%$ Faulbolz.

III. 1i. - Ein tapijober iogenannter tranfer Boden. - Së̈he 735-749 m. Sage und Boben: F̧aft eben. 3äher weiker, grauer lettiger

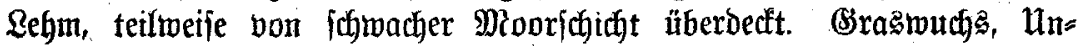

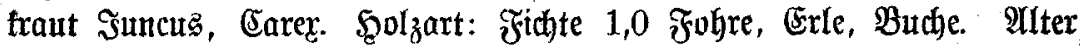

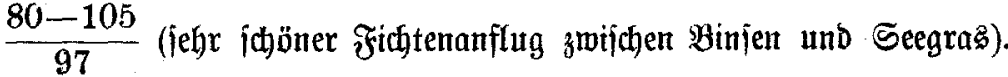

\$on $2215 \mathrm{fm}$ (meift $\mathfrak{B i n b m u r f ) ~} 98 \mathrm{fm}$ faul $=4,5 \%$ Faullgolz.

VI. 3 h. Şöhe $712 \mathrm{~m}$. Rage und Boden: Eben. Teils lettiger \$oden, größ̄tenteils aber Moorbobell, zum Teil übergangômoor. Sphag= num, Equifetum. Şolzart: Fithte 1,0. Alter $\frac{70+140}{95}$.

Bon $333 \mathrm{fm} 15 \mathrm{fm}$ faul $=4 \%$ Faulhol . 
2. Şartb̈̈ben.

IV. 3a. Şähe 700-724 m. Sage und Boden: Biemlich exponiert; Sartboden jandiger Rehm. Fithte 0,97, Tanne, Buche, Fohre, Ahurm, Ulter $122+136=131$.

Bon $2516 \mathrm{fm} 387 \mathrm{fm}$ faul $=15 \%$ Faulholz.

IV. 5a. Scähe $733-750 \mathrm{~m}$. Rage und Boben: Fajp eben; jehr

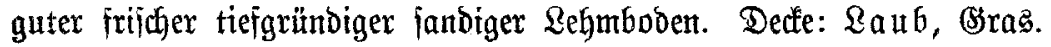

Bon $2834 \mathrm{fm} 299 \mathrm{fm}$ faul $=10,2 \%$ Faulbolz.

IV. 6e. Söhe 725-735 m. Lage und Boben: Fraít eben; fehr guter friicher Rebmboben. Decte: Laub, Bras: Ggolzart: Fichte 0,94, Buthe 0,06, 战ler 127.

Bon $4235 \mathrm{fm} 539 \mathrm{fm}$ faul $=12,7 \%$ F̧aulfolz.

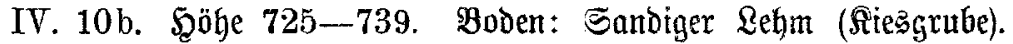

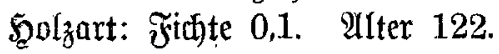

: $\mathrm{on} 754 \mathrm{fm} 132 \mathrm{fm}$ faul $=17.5 \%$ Faulfydz.

YY

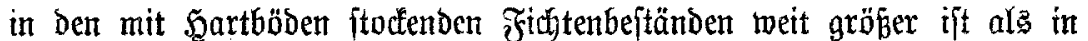

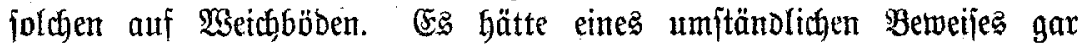

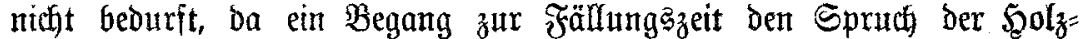

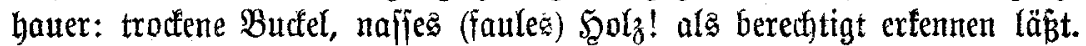
Die Worte bes Altmeifter anormalen Standortagebiete vermehren iinh bie Bebrohungen ber Firfte burch Rotfäule", permag ich aljo nicht für berechtigt zu halten, ba bie Scartböben, welche durchaus erifflajifge Fichtenftandorte find, wohl von niemand ben anormalen Stahborten zugemiejen werden.

Die Ergebuifje ber amtlich geführten Faulfodziftatifitif, welche ber gegenteiligen 2 lilifauung ber Forjteinrtrhtungareferenten und bes Wirt=

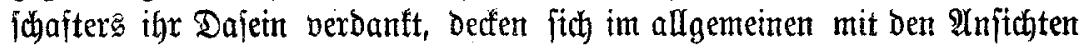

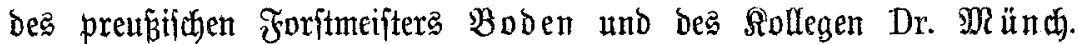

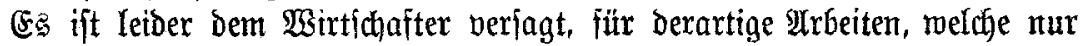
jo nebenbei zu machen find, jene Beit aufzumenden, die Beriuche auf

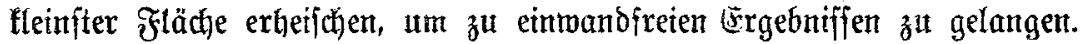

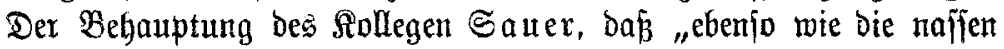
Nieberungen zumeift auch bie anmoorigen Ragen, bie bod bes Sticfitoffs

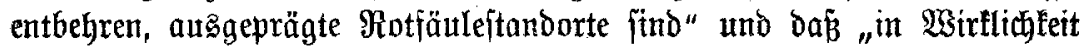
jïch Die Rotfäule befanntlich in beionderent Maje in jolchen Mulden uno Nieberungen findet" vermag ich, auch wern fite von Wirtichaftern aus Den veridhtedeniten $\mathfrak{B u c h s g e b t e t e n ~ - ~ D b e r f r a ̈ n f i t c h e n ~ S a r a , ~ \Re u ̈ r n = ~}$

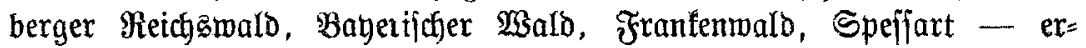
härtet wiro, auj (Srund meimer Erffabrung ứ Statiftif nicht beiźzutreten. 
Die hier oft wahrnegmbare Ericheinuntg, baj jith bie Fichten an ben Scodjmoorrändern, die außser Spirfen (Pinus montana) höcjitenz Birfer aujweijen, zu ganż anjebulitchen Mafjen entwicteln tann uno fith bort

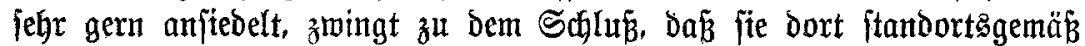

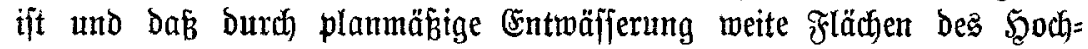
moors jür die forjtlide Benubung tauglich gemad)t werben fömntent.

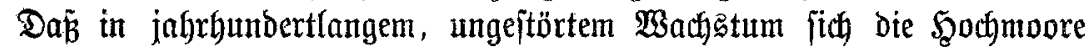

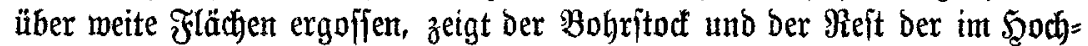
moor untergetauthten Bejtofung von Fichte, Tanne, felbjt Burche uno Eibe.

Menn Sollege Sauter fohreibt: "Es ijt allgemein befannt, baj ber Rotfäulepilz bon einem Serbe aus ftch) zentrifugal verbreitet (jogenannte

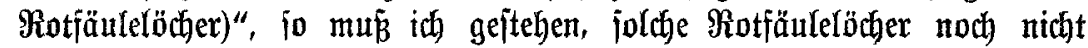
beobachtet zut haben, weder auf Şart= noch auf $\mathfrak{B}$ eichböbern. Daß́ in

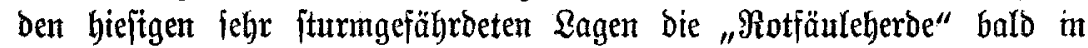
Eridbeinung treten mußsten, bedari feiner Worte, da $\mathfrak{B}$ bei ber Stod $=$ züblung auf Siablhieben, wo 50\% Der Stödfe fitch al: faul erwiejen, eine gruppenweife Ragerung nidht überiegent werben fonte, Itegt auf ber

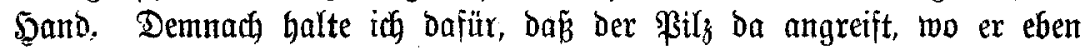
Wachstumbbebingungen finbet.

Die Statiftil ift audy geeignet, Slufficlizffe barïber zu geben, ob bie Buche bas bejte Seilmittel gegen bie zunehmende Bsefahr ber Rotfäule - Sauer'a Arbhanblung 5.24 - ift.

Bunächit 4 Bejtänbe, in melchen Bưḑen nicht borfommen.

IV. $9 \mathrm{~d}$ von $657 \mathrm{fm} 68 \mathrm{fm}$ faul $=13 \%$ Faulfolz

IV. $13 \mathrm{c} " 681,72, "=10,7 \%$ "

IV. $14 \mathrm{c} " 2653$ " $268 "$ " " $=10,1$ " "

IV. $14 \mathrm{~d} " 1896$ " $243, "=12,8$ " "

oder in Sutmma der 4 Bejtänto (\$artboden)

von $5897 \mathrm{fm} 651 \mathrm{fm}$ faul $=11,6 \%$ Faullhold.

Diejen werden gegenïbergeftellt bie ictjon oben genannten Beitänthe.

IV. 5a mit $3 \%$ Budben von $2834 \mathrm{fm} 299 \mathrm{fm}$ jaul $=10,2 \%$

IV. $6 \mathrm{c} " 6$ "

in Summa $7069 " 838 "$ " $\quad 4235=11,8 \%$

Dann nod) Die Bejtände im Diftrift $\nabla$ Grfrallen, welche neben Fithten aud Tamen, Buthen; (Ej)hen, Ulmen, athorn, Erlen enthalten. V. 1 e $24 \% 140$ jäbr. Bu. von $659 \mathrm{fm} 99 \mathrm{fm}$ fautl $=15 \%$ Faullholz V. $2 \mathrm{~d} 9,145, " \quad 3067$ " $422, "=13,7 \%$ " " $\nabla .3 \mathrm{a} 3$ " 130 " " 773 " $117 "=15$ " "

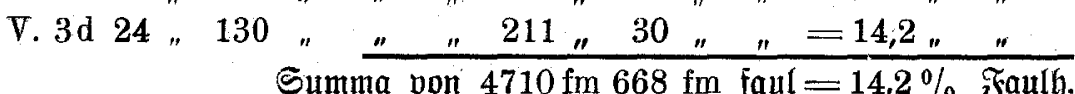




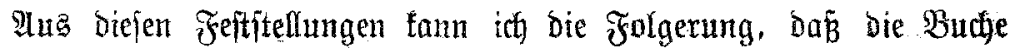

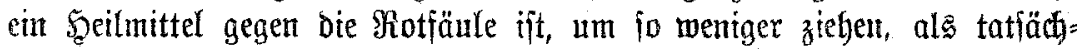

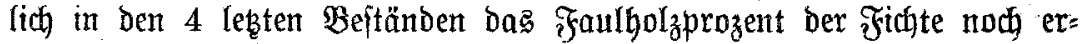
beblicher ijt - V. 2 e mit $24 \%$ Butben und $31 \%$ Faullyolz wurbe

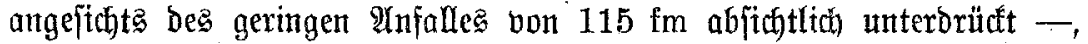
weil auch bas Tamnentholz unter bem (Gejamtanfall von $4710 \mathrm{fm}$ in begriffen ift. Das bejtodungaproz̧ent ber Buche bez̧ieht fith nux auf

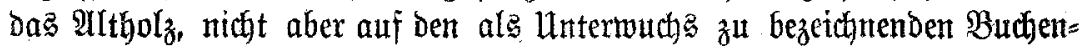

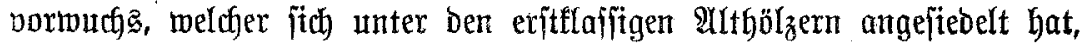
aber bei ber Fällung größ̄tenteils zugrunde geht. (Fine günftinge Mirfung

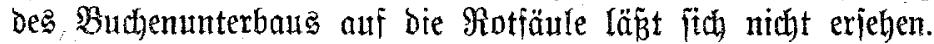

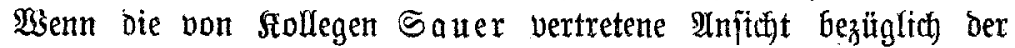
Bucfenbeimijofung ridjtig ifit, jollte bie Fichte, wenn fie eimzeln ober in (Bruppen in \$uchenbeitänden iteht, wentg ober gar nicht itočffaul feir.

IV. 5d. 80-104jähr. Buche 0,51, Fichte 0,49. Miichung einzeln, WSudjs fefyr gut.

Bon $167 \mathrm{fm}$ Madelgolz $30 \mathrm{fm}$ faul $=18 \%$.

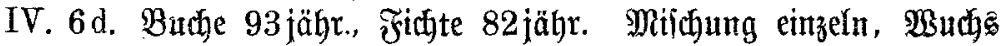
iefric gut.

Bon $188 \mathrm{fm} 35 \mathrm{fm}$ faul $=18,6 \%$.

Die Statị̂tif zeigt, Daß in Buchenbeftänden dą Faulfyolzprozent ber Fichte nicht fleiner fondern größ̈er ift; Demnach barf angenommen

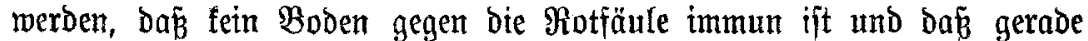
bie gutburchlüfteten Böben Dem $\mathfrak{B a c h s t u m}$ Der Trametes nicht weniger gitnfitig find als jenem Der Fichte.

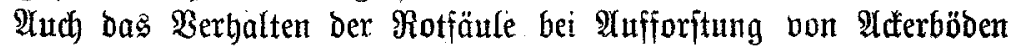

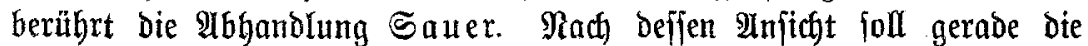
1. Generation unter ber Rotjäule mehr letben als bie folgenden. Setber unterbleibt näbere Begrünoung; , fie wäre zmeiffellos iegr intereffant, da Sauer S. 23 fagt: "bereits die zmeite Beneration - nach Raubholz -

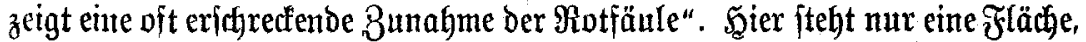

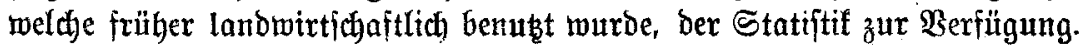

IV. 11 b. 24,2 ha. \$öbe 700-741 m. Booden: 〔andiger \&ehm,

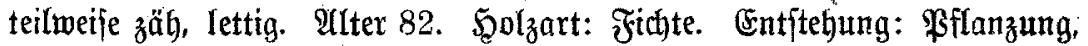
2,40 m Reibenpflanzung, erfter Waldbau mit ehemaligem Feldboben; $\mathfrak{B a u c h s}$ jebr gut.

Bon $1267 \mathrm{fm} 65 \mathrm{fm}$ faul $=5 \%$.

(5) fteht fomit Das Faulbolzprozent bes ehemaligen OAdferbodens noch unter bem Durdijhntte Des auf $\mathfrak{B e i h b o d e n ~ e r m i t t e l t e n ~ - ~ 7 , 1 ~ - ~}$ uno ift taum bie \$älfte jo groß̧ wie jenes auf Şartböben - 12,7 - 


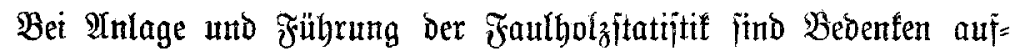

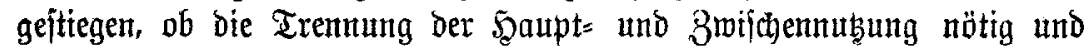
berechtigt ijt. Da boch Der Bwect Der Statifiti in Der Feftitellung liegt,

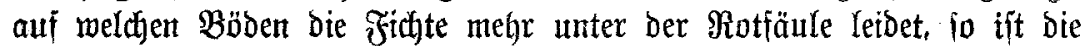

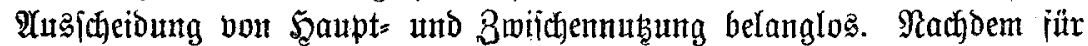
bie hiefigen $\mathfrak{B}$ erhältniffe ein Urteil fajon auf Brund Der Erfahrung ge= jällt werben fonnte und biejes burch die Statifitif erbä̀rtet wirb, fyalte id e马 für notwendig, bie Unteriuthungen örtlich zu beichränfen, aber imiv=

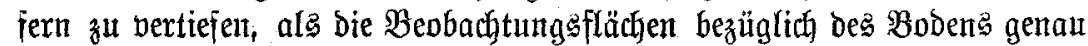

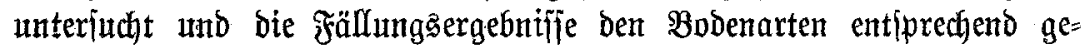
trennt gefalten werben. So wirb man in \&agen, wie ben biefïgen, wo Sgart= und $\mathfrak{B e i c h b o ̈ b e n ~ v f t ~ i n e i n a n b e r ~ u ̈ b e r g e h e n , ~ a m ~ e b e i t e n ~ z u m ~ B i e l e ~}$ gelangen. Daj eine geringe Moorïberlagerung weder bas Wadjstum ber Fidfte noch jenes der Trametes weientlich beeinfluffen fann, Dürfte faum anzuzweifeln fein uno es fönnten jitch bie Bodenumteriuthungen in jenten (Srenzen balten, bie aud) bem Wirtjchafter bie Mitarbeit nodh geftatten.

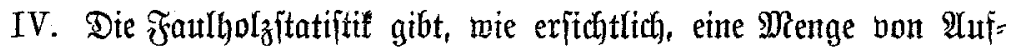
idulüffen und lohnt jo bie aufgemendete Miäbe.

Bur Stellungnafine zu ben von Rollegen Sauer behandelten boer

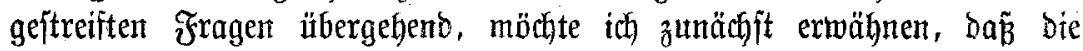
Wurzelbitbung Der Fichte auf Moorweichböben eine andere î́t als auf Den tiefgrüntotgen Scartböben. Huf Dem Moor fommt bie flache $\mathfrak{B e}=$ wurzelung Der Fichte am bejten zitr Beltung. Warzalballen von faum $20 \mathrm{~cm}$ Dicfe und 30 und mebr Sutabratmeter Fläche find nichto $\mathfrak{A} u \hat{\beta} e r=$

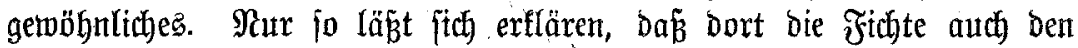
ftörfften Wimben ftandzuhalten bermag und ich ftehe nicht an zu

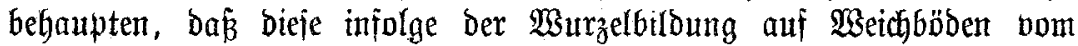
Sturmmind mentiger gefährbet ift afs auf Şartböben. WUf folchen gehen bie $\mathfrak{B u r}_{3}$ eln mehr in ben Boben, laden nidft foweit aus und treiben weniger Seitentwurzeln; Der $\mathfrak{B u r z}$ elfitod ericheint hier fompafter. Begen

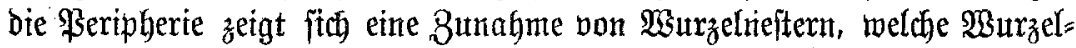
fajent nad) Der Tiefe fenden. Der Scolzbauer nennt bieie WSurzelnefter "Taben". Dieje finden fich im Scart= wie in Weichboden, find aber hier

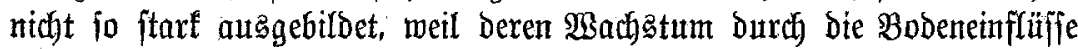
gehemmt ift. Die Tabenbilloung - bie Befägigung an einzelnen Stellen

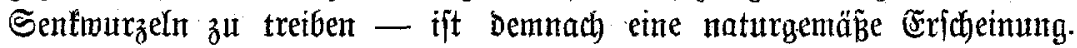
Die Fichtenmurzel fentt fich in ben Boben, um bort einen Stügpunft za geminnen, foweit als fie barin zu leben vermag.

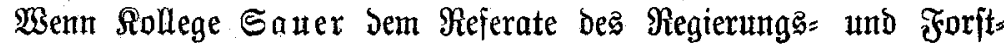




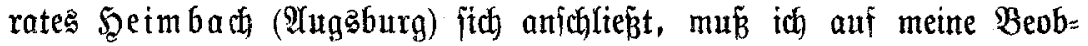

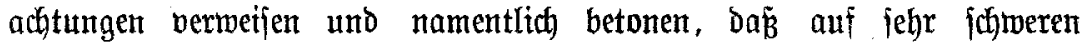

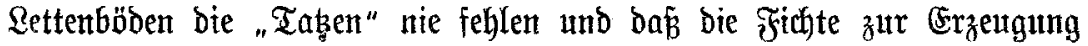

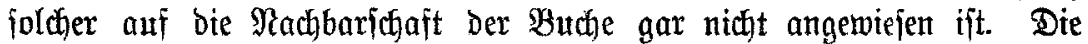

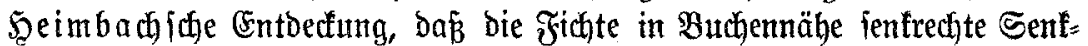
murazeln nach ber Tiefe fenbet, it jomit ein allgemeiner Yorgang, ber burch Stodtrobungen an anderen Stellen Beitätigung gefunden fjätte.

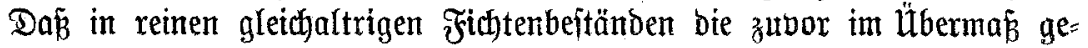

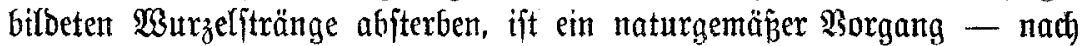
den Forichungen bes Frofeffors Frant $\subseteq$ brara fterben bie Saug= wurzeln ber $\mathfrak{g}$ olzpflanzen ab, twenn fte ben $\mathfrak{B o d e n}$ ausgenubzt haben -

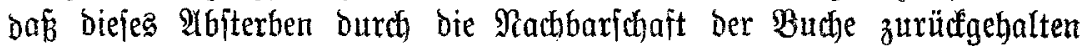
wiro, ift nur Innahme uno nicht betwiejen. Die oben mitgeteilten $\mathbb{Z} a \mathfrak{b l e n}$

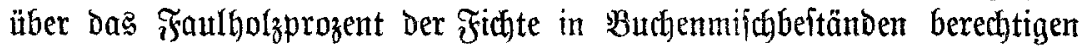

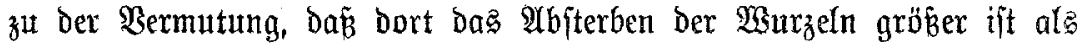
in reinen Fichtenbejtänben. (E) ift auch idhroer zu begreifen, wie auf

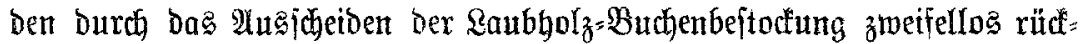

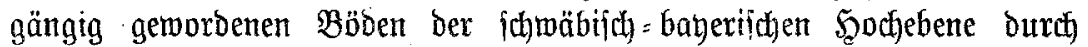
mehrere (Senterationen eritflaffige Fichtenbejtänbe ermachien fonnten, nact)=

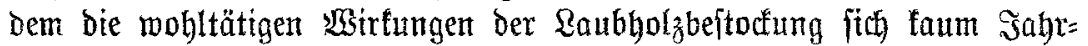
zehntelang jühlbar machen fann.

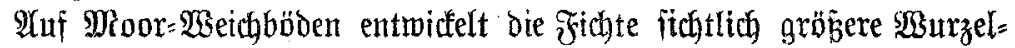
maffen; jie muñ jïch fefter veranfern und bie Räbritoffe aus weiterer

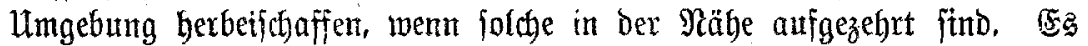

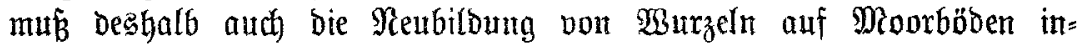

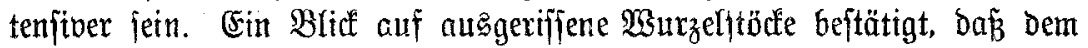

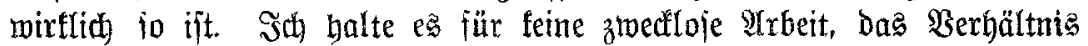

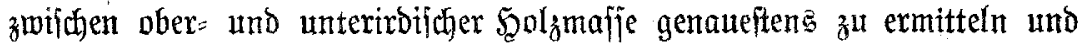

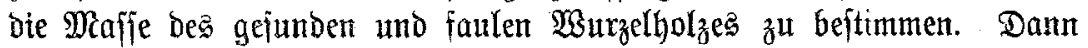

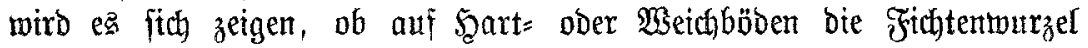
mehr burch Fäulnie leibet.

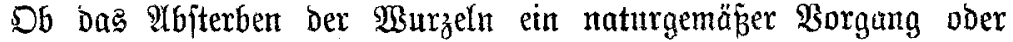
nebenbet eine Sranfheitsericheinung bes alltere unb wie weit bie Tramete baran betelligt ift, find nod offene Fragen; fejtitehen bitrfte, Dấ ent=

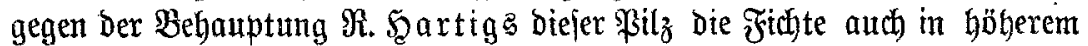

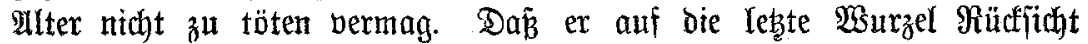
nehmen und plöblich, wenn er vier Fïnftel der Şolzmaffe zerjtört hat, bie firaft verlieren follte, Den Rejt Der Wurzel zu Durdobringen, vermag

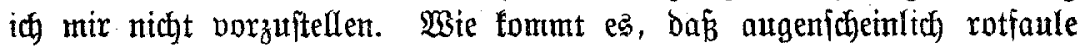
Fichten alljährlich neue Triebe bilden, normalen Zumachs liefern und 
Sahrzebnte lang des bejten Waahstums fth erfreun? Wsem die

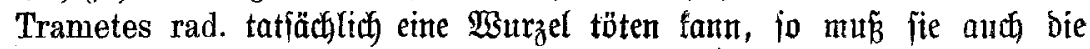
Ieşte unb Damit ben Baum felbit töten tönmen. Sch habe bieien Falf nodi) nie beobachtet.

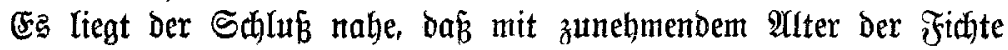

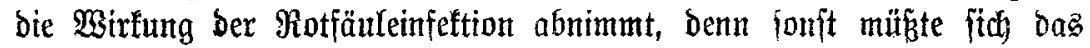
Bill einer von biejer getöteten $\mathfrak{F}$ ichte recht oft bieten. Demnadj vermag

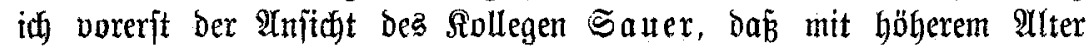
Der Brab und Umfang der Berfeţung zunimmt, nicht beizutreten, weil Defijen wettere Bermutung, daß̧ ein im 50,-60. Sebensjahr noch ge=

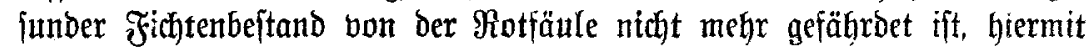
im Biberiprud iteht.

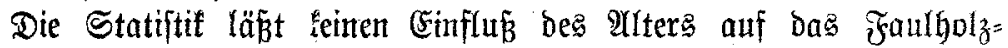
prozent exfennen. Mertwïrbig tït, bå̉ gerabe jene Stämme, welche aứ ber Sonnittfläche mur mehr eine ifywadhe Splintidjicht zeigen, fich am idfuelliten gejund fonneiben und io am wenigiten Fautlfolz liefern.

Sillege Sauer glaubt, baj ber Inthlicf einer von Tram. rad. ge=

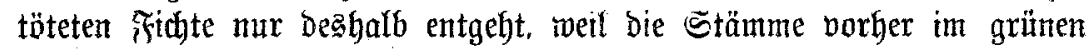
Buitande nom Winde gemorfen merben, wern die Şauptwurzeln vom \$itze zeritört find. Sst dem bieftgen, vom Sturmmino off und ichwer heingejuchten Finftengebiet miro man nur wenige völig umgettürzte

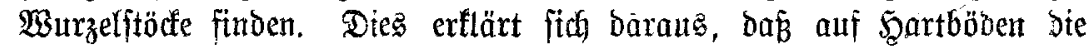

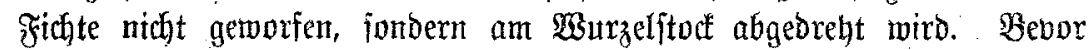
noch ber Stodf mit bem anbängenden Rebmballen herauggeriffen mird,

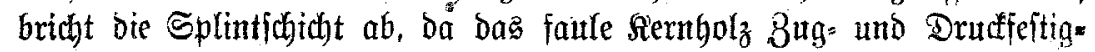
feit verioren hat. Şter zeigt fith Der Unterfajied der Böben haaridarf; im Şartbodent mehr $\mathfrak{W}$ inbowurf, b̀e Stämme abgeoreht; im Wheidbobent weniger Wintomurf, die Stämme nicht gebrochen, fọtoern umgefippt.

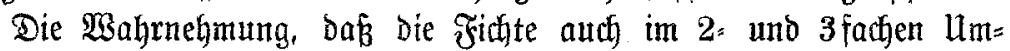

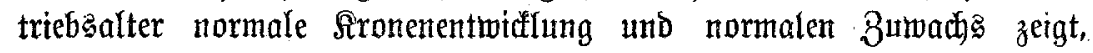

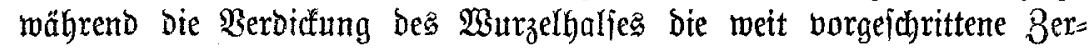

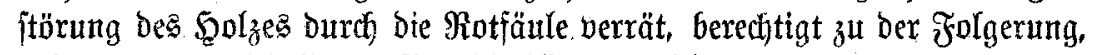

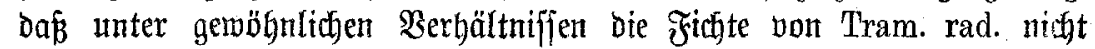

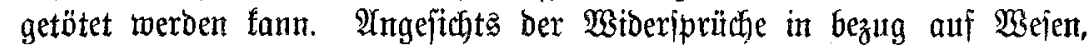

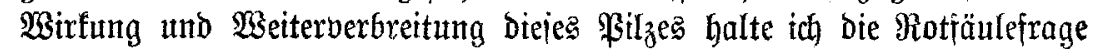

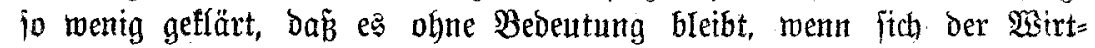

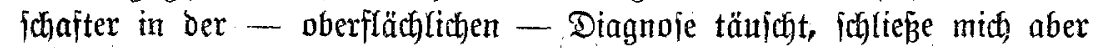

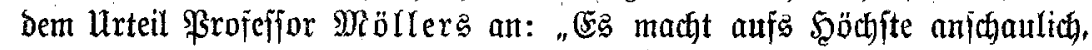
Daß̧ bejtimmte, bisher unbefannte Bedingungen bazu fommen müffen,

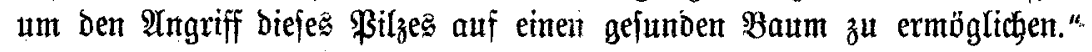


V. Wann und wie ber Ilngriff ber Trametes rud. eriolgt, ijt nod

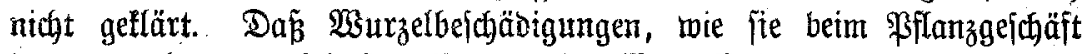
tmmer vorfonmen, bei Zerreiß̧ungen ber Wurzeljtränge beim Sturmmind

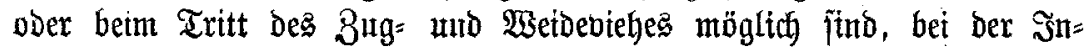
feftion eine nidjt untergeoronete Rolle iptelen mögen, ift benfbar, und wirb

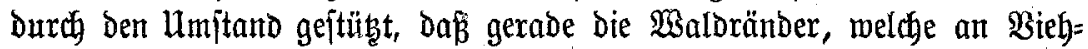
meiben ftoßen, am meiften rotfaul fino. Sntwietweit bie Märme= Sonnen= beftrablung bierbet föroernd in Frage fommt, foll nicht näber unterjucht werben, es genügt bie Feftftellung, Dấ gerabe Siübränder auffallend rot=

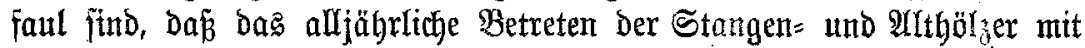
Zugvieh zwedts Şolzabfuhr weder für den Boden nod für Den Bejtand

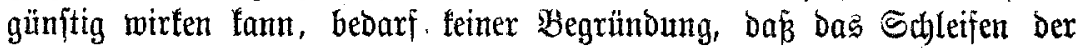
Stämme während der Sommermonate mit zahllojen Wurzelbejchäbigungen

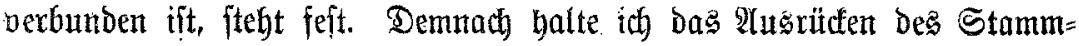

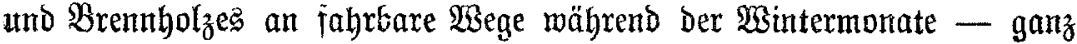
abgejejen bon peluntären Borteilen - jür ein herborragendes mittel, un Boben und Beftand gejund zu erbalten. (5) genügt Darauf bingu=

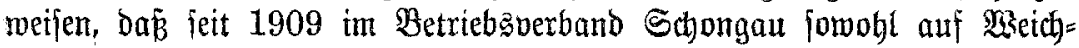

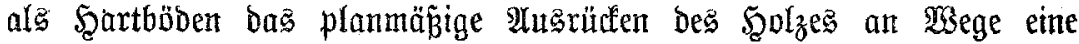

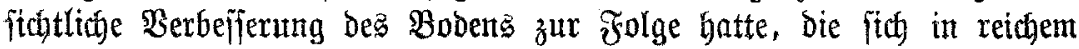

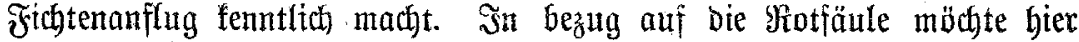
feitgeitellt werden, Da $\bar{B}$ in jenen (Bemeindemaldungen, wo bant ber $Z_{\mathfrak{a b l}}$

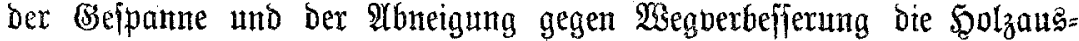
bringung immer mäbrend ber $\mathfrak{B i n t e r m o n a t e}$ erfolgte, bas Faulbolz= prozent laum nermensmert ijt, obgleich dort bie Beitände eber älter find, als in ben angrenzenden Staatâmaldungen bes Sadjentrieder unb Dien=

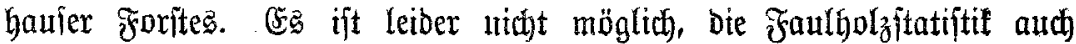

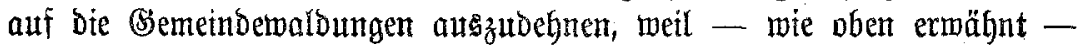
Das Prozentuerbältnies Der Tanne zur Fithte ein andere ijt und bielfach mechjelt; in den Staat waldoungen bes Betriebsuerbandes Sthongau $1 \%$

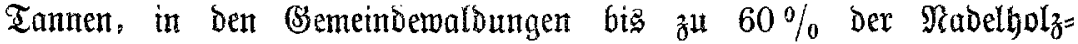
beftocitung.

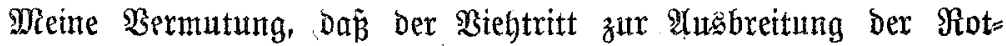
fäule beiträgt, wiro burch bie $\mathfrak{B}$ enbachtungen jener Sollegen geftüb̧t, welche betonen, Daßj Die Rotjäule in jüngeren WSalobeftänden, Die mit Wetbeberechtigungen belaftet find, auffallend un fith greift.

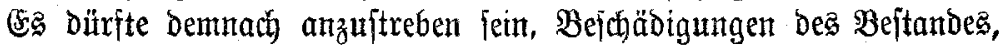

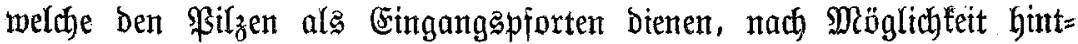
anzuhalten und das $\mathfrak{S c o l}_{z}$ während der $\mathfrak{W i n t e r m o n a t e ~ a n ~ b i e ~} \mathfrak{W}$ ege zu

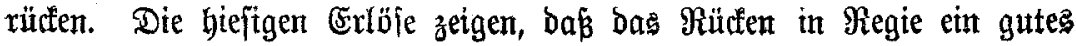




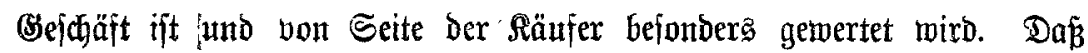

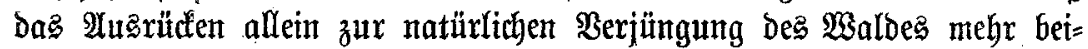
trägt als andere forjtlitche SMaknahmen, beftätigt mir bie hter gemonnene (srfabrung. Wenn bei $\mathfrak{A} u f f t e l l u n g$ ber Grumblagen für bie Foriftein=

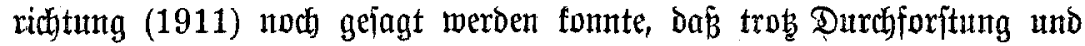
Blänterung bie Naturberjüngung fịch mur ipärfich zeige, fo vermag heute

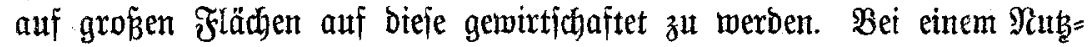

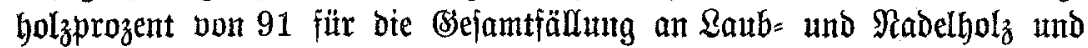
einem jährlichen $\mathfrak{2}$ nfall von ca. $1000 \mathrm{fm} 2$ und $3 \mathrm{~m}$ langen Riften= hölzern blieb meines (5rachtens dem Wirtichafter nur bie Wabl, auf bie natürliche ßerjüngung zu vergichten ober für bie Bringung böhere $\mathfrak{A} u \mathfrak{l}=$

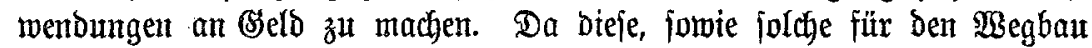
mieder Durch bie bögeren Erlöfe wettgemadht werben, jo fann id auf

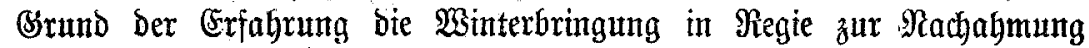
empiehlen, zumal dann auth die jüngften Schläge in Bejtodłung gebracht werben tönnen. Daf ex trob der geringen SBeimijoung von Butben auf

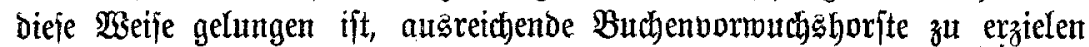
und Damit ben fünitlichen Borbau von Buthen ummötig zu machen, Dürfte jenem $\mathfrak{B e r f a h b r e n ~ f i t h e r ~ F r e u n d e ~ g e t w i n n e n . ~}$

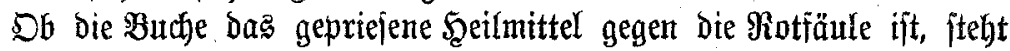
Dahin; es märe wümidjeng̊mert, wenn von Seite ber Mirtfchafter Material herbeigebractst mürde, um bieje Frage zu löjen, nachdem meine Errhebungen als auf zu beịhränftem (Siebiete gemadht, untzulänglitch findo, um ein $\mathfrak{U} r=$

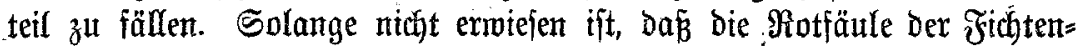
beitärtbe butch Butchenbeintijhung eingebämmt werden fann, fann th mich mit bem $\mathfrak{B u t h e n d o r}=$ ober =unterbat nicht befreunden, zumal beide eine ftarfe Durchlichtung Des Fichlenbejtandes exfordern unb biejen für Den Bindwurf reif machen. Nach meiner Erfábrang entwiçelt bie Fichte im reinen Bejtande nicft nur bie größ̈ten Majien, jonbern auch bie beften Stammiormen. Die Beiniijuhung von nur etlichen Brozent Butchen brüdt bie Majien = und $\mathfrak{B e r t p r o d u f t i o n}$ bedeutend, obne bierfür auth nur einen Borteil zu gemäbren. Nie und nimmer mebr tann bie Buthe, Der es nicht att Rahrung wohl aber an Waärme gebricht, mit ber Fichte in Deren Seeimatgebiet fonfurrieren und bie verichtedenften Billber auts ber

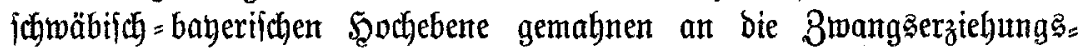

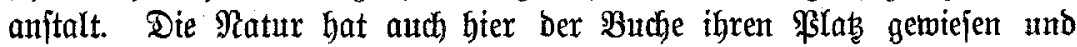
Dả jind bie jüblichen (singänge zat Den Bräben uno Sdhludhten (Tobel); Dort finbet fie alles was fie brautht, auth unbeftrittenten Sronenraum. Die natürlide Berjüngung aflein vermag ber $\mathfrak{B u c h e ~ n i c h t ~ m u r ~ b i e ~}$

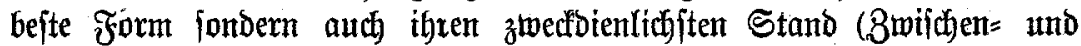




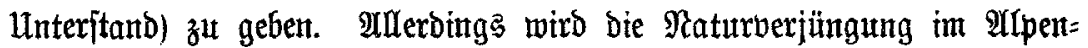
vorlandgebiet burch) Den ungemein ftarfent (Srastwuths und bie Dimenfionen bez Fichtenlangholzes erichtwert, inbem bas Bras, ben Waagenfpurent folgenb, allmählith bie garze Flöche ïherziebt unb bie Bringung ber

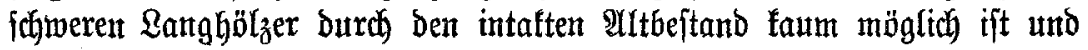

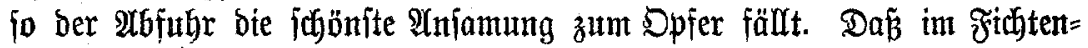

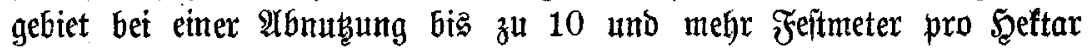
bie räumliche Binountg ber Wirtfichaft Durch ben auf ein Dezennium feit= gelegten Fällungaplan redft oft bie naturtwibrige Berjüngung smethobe

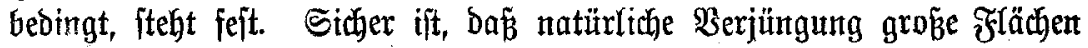

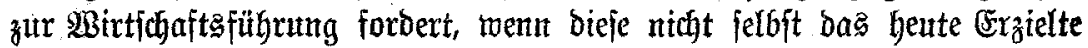

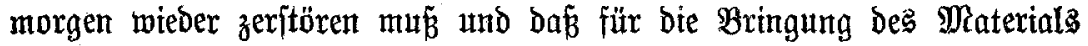

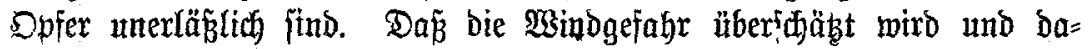

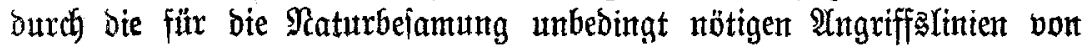
Şaus aus zu fnapp bemejien werten, bedarf feiner Worte.

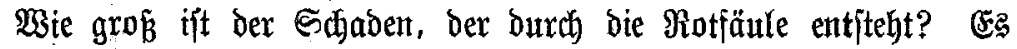

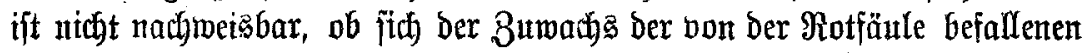

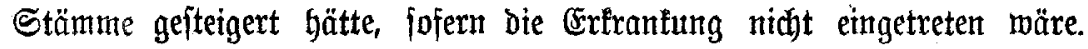

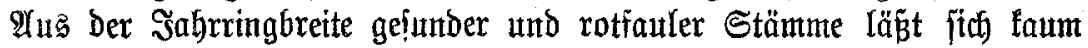

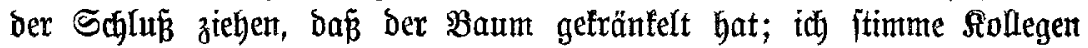
Sauer völftg zu, Dẩ bie Firone erfranlter Sndivibuen fein Merfmal Der Erfrantung zetgt; Demnach mut

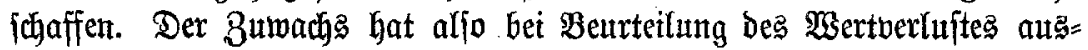
zaticheiben; es ift nur ber Berluft an geiundem Sgolz zut buchen. 2Allex=

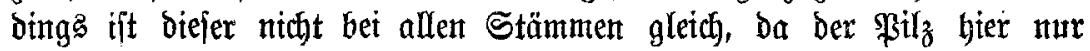
2-3 m, bort 8-10 m hoch ftetgt; es fann bemnach burch bas $\mathfrak{A} b=$

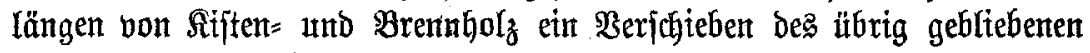
Stamme in bie niebrigeren Sortimente itattfinden.

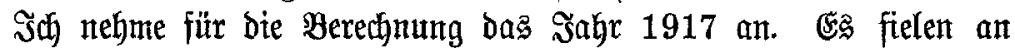
$902 \mathrm{fm}$ Rijtenholz und $218 \mathrm{fm}=311$ Ster fautes unt anbrïchiges Brenntyolz, in Summa $1120 \mathrm{fm}$. Dieje würden, wenn geiund, burch $=$ ichnittlity Dex II. Rlaffe \&angholz angehören.

$1120 \mathrm{fm} \mathfrak{R} \mathfrak{a}$ angholz II. Silafje à $63,50 \mathscr{A} \quad$. . . . $=71120 \mathscr{M}$

Errlöft wurben att

$902 \mathrm{fm}$ Riftentbolz à $36 \mathscr{A l}$. . . $=32.472 \mathscr{H}$

218 "311 Ster Brentuholz à $6 \mathscr{H}=1866$

$34338 \cdot \frac{\mathscr{a b} 34338 \mathscr{H}}{36782}$

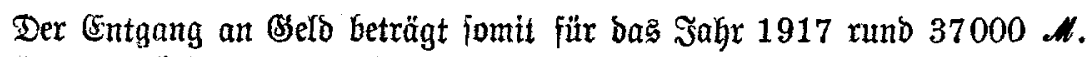

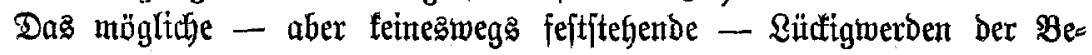


ftände fteigert Die Bornubsungen, erbäht bamit bie $\mathfrak{B e r z i n j u n g ~ u n d ~ b a u r f t ~}$ nicht bejonders bemertet zut merber.

Der Zwed biefer Arbhandlung, weldbe fith burch alle Fächer bes Schreibtifches boch mieder and Tageslicht arbeitete, ift exfüllt, wem bie

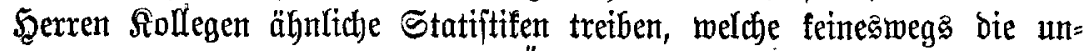
frudbtbarften bleiben, fofern fite ber D̈ffentlichfeit nidft vorenthaltent werden.

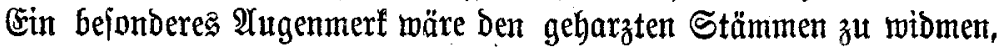
benn bieje jollen am ehejten rotjaul werben. Nadj meinen Beobadtungen fơreitet bie Trametes in ben vor 50-60 Safren gepechelten Stämmen lange nicht in Der Beife in bie Şöhe, mie an ungepechelten Stämmen.

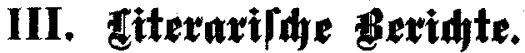

Mr. 14.

\section{Die Berbecitung ber foxiflid widjtigen Bäıute utb Stxäudex}

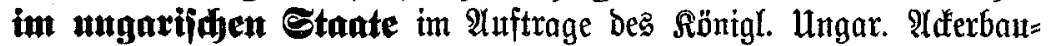
mintifteriums mit Benub̨ung ber unter Dex Reitung Des Minijterialrats, Sedtichulprofeffors i. \$. Rubwig Fefete vorgenommenen forjtlichen pflanzengeographifhen (Erbebungen berfap̧t von $\mathbb{R}$ ubitg Fefete und Tibor Blattny. Mit 5 farbigen fiarten, 18 fiuntheilagen in Selid=

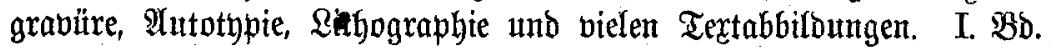
Scerauzgegeben vom töntigl. ungar. Minifterium fïr $\mathfrak{A}$ (ferbau. Sefmec=

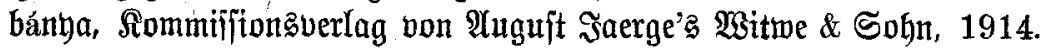
(II. BD.: Tabellen.)

Ulm 7. September 1914 follte in Bubapejt bie VII. Beriammlung

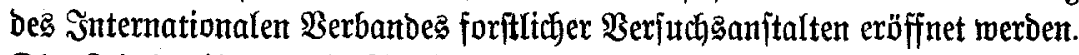

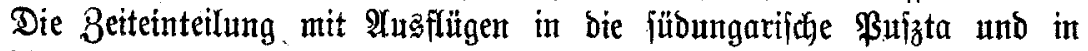
bie hohe Tatra veriprach aukerorbentlich viel, aber alles wurbe Durch Den Sriegsautbruch vereitelt. Zu Den zahlreichen Druffichriften, die Den zur Tetlnahme 2Atgemeldeten bereits im Sulf 1914 zugeạangen waren, fommt nun noch das oben genannte ftattliche Werf binzu, Dag gleid $=$ zeitig in ungarificher und beuticher Sprache erichien und Den Teilnehmern ber Serjammlung überretdyt werden jollte. Die bentiche l̈berjebsung ift vom ungarifiden Dberforftingenieur und 2tbjunften ber Zentralveriuch

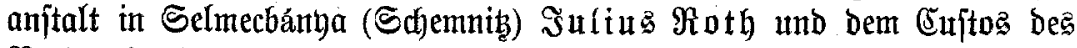

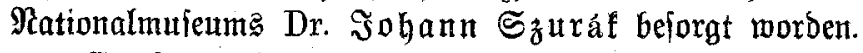

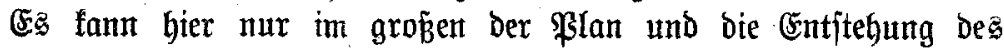
vorbilblichen $\mathfrak{W}$ erfę 